\title{
Empirical Research on Memetic Transmission and Adaptation of Media Language
}

\author{
Y.X. SONG \& J.Wei \\ Jilin University, China \\ M. Yang \\ Jilin University, China
}

\begin{abstract}
Media language has become more and more prominent and powerful in this highly-developing information era. Taking memetic adaptation theory as the rationale, this paper explores the characteristics and classifications of the media language memes and investigates their memetic adaptation in transmission by collecting and analyzing different types of memes in a variety of media platforms. It is concluded that the transmission and replication of media language memes are a process of choice and adaptation. Contextual correlates (e.g. mental world, social world) are what media language memes adapt to in the process of their transmission and replication.
\end{abstract}

KEYWORD: memes; adaptation; media language

\section{GENERAL INSTRUCTIONS}

Media language, an umbrella term including all languages employed in a variety of media platforms, becomes more and more prominent and powerful in this highly-developing information era. It reflects hot issues on economy, politics, science and social life of the modern society and technical developments accelerate the production and evolution of media language, especially its transmission (Wang 2013). Therefore, it is significant to make a detailed exploration on media language transmission. Many researchers have noticed that media language is imitated, replicated, and adapted by a large number of people to transmit information (e.g. Aitchison and Lewis 2003, Liu 2007 among others). In other words, media language is featured by its memetic transmission and adaptation.

\section{MEMETICS AND ADAPTATION THEORIES}

\subsection{Memes and Memetics}

The concept "meme", introduced by Dawkins (1976), is defined as "an element of culture that may be considered to be passed on by non-genetic means, esp. imitation" (Moritz, 1990). Its original definition by Dawkins (1976) is constructed in terms of replicator as he argues that evolution does not depend on gene----the particular chemical basis of genetics; however, it relies on the existence of meme, i.e. a self-replicating unit of transmission
(Dawkin, 1976). Blackmore (2000) further explains the concept of meme from the perspective of individuals: memes are "whatever is copied from one person to another person, whether habits, skills, songs, stories, or any other kind of information" (Blackmore 2000, p52).

Based on the abovementioned definitions, the concrete forms of memes have been explored. For instance, Atran (2001) puts forward that "candidate memes include a word, a sentence, a thought, a belief, a melody, a scientific theory, an equation, a philosophical puzzle, a religious ritual, a political ideology, an agricultural practice, a fashion, a dance, a poem, a recipe for a meal, table manners, court etiquette, or plans for cars, computers, and cellphones" (Ibid. P351-381).

Memetics, a theory based on the concept "meme," have aroused a wide concern in the academic circles and is depicted as "an approach to evolutionary models of cultural information transfer" (Brodie 1996, P80). Memetics has also been adopted to analyze information transmission and linguistic phenomena by many researchers (e.g. Heath and Seidel 2004, Moritz 1990, liu 2007 among others).

\subsection{Adaptation Theory}

Adaptation theory, proposed by Verschueren (2000), focuses on linguistic and context structures at different degree of consciousness in language use (Wang, 2013). According to Verschueren (2000), the use of language is just a process of continuous 
linguistic choice-making. Three hierarchically related concepts i.e. variability, negotiability and adaptability are paramount to understand the process of "making choices" and adaptability is the core of adaptation theory.

Variability is "the property of language which defines the range of possibilities from which choices can be made" (Verschueren, 2000: 59). It indicates that a variety of options are accessible to language users as they transmit information.

Negotiability refers to "the property of language responsible for the fact that choices are not made mechanically or according to strict rules or fixed form-function relationships, but rather on the basis of highly flexible principles and strategies" (Verschueren, 2000: 59). Each choice of language users derives from a different consideration and hence makes a different effect. In other words, language users make a choice among a great number of expressions in accordance with the context and/or the communicators.

Adaptability then is defined as "the property of language which enables human beings to make negotiable linguistic choices from a variable range of possibilities in such a way as to approach points of satisfaction for communicative needs" (Verschueren, 2000: P59). This property makes language one of most important tools for human life and survival.

In summary, adaptation theory underlines that "language use serves the purpose of communication and the choice of language is subject to the concrete context" (Liu 2007)

\subsection{Four Angles of Linguistic Adaptation investigation}

From Verschueren's point of view, the linguistic adaptation can be explored from the following four angles: 1) contextual correlates of adaptability, 2) structural objects of adaptability, 3) dynamics of adaptability, 4) salience of the adaptation process (Verschueren, 2000: 63). These four angles are in perfect agreement with the linguistic, social, cultural and cognitive elements involved in the dynamic process of language using.

Contextual correlates of adaptability includes all the extra-linguistic ingredients of the communicative context with which linguistic choices have to be interadaptable; structural objects of adaptability refers to any layer or any level of linguistic structure, from sound feature to discourse or text; dynamics of adaptability is the unfolding of adaptive process in interaction; and the salience of the adaptation process means the degree of conscious when choices are made (Versehueren, 2000: P63). These four angles of investigation relate to each other and they play an important role in the research.

\section{RESEARCH METHODOLOGY}

This study carries out an investigation of the qualitative analysis, aiming at building a pragmatic model to analyze the transmission of media language on the basis of Linguistic Adaptation Theory and Memetics.

\subsection{Data Collection}

Data in this research are chosen from media platforms, for example, Chinese and English newspapers, TVs and movies, Internet community forums etc. These data are representative because these media transmission channels play an important role in the mass media and a great amount of popular language is spread largely through these channels nowadays. Secondly, people's acceptance of memes generally comes from the universal use on the mass media, thereby analyzing data collected from them is reliable and valid.

To be more specific, 200 data of memetic adaptation have been collected from Chinese and English newspapers, movies and TV subtitles, and the Internet. For example:

(1) All roads lead to Venice (Newsweek 05, Sep, 2012 P9)

(2) Trinke: What are your intentions?

Brian: Huh?

Trinke: What are your intentions with my daughter? Do you plan on marrying her?

Brian: I don't know.

Trinke: Well, only married girls and boys... Ahem...Show each other their, uh...parts.

A few days later, Gertie and Maya have the following conversation:

Gertie: What are your intentions?

Maya: Excuse me?

Gertie: What are your intentions with my father? Like, do you plan on marrying him?

Maya: Uh... I don't ...

Gertie: Because I was taught that only married girls show their girl parts to boys.

Gertie: Right, Daddy?

(from movie Jersey Girl)

(3) No zuo no die (From the Internet)

(If you don't do silly things, they won't come back and bite you in the ass)

In summary, language in all media platforms has been explored and typical language memes have been collected to improve the validity and reliability of the study.

\subsection{Data Analysis}

After collecting media language materials, which seemed to be unsystematic, some typical features of media language memes and their transmission have 
been analyzed. Three steps have been taken in the analysis procedure.

The first step was to conduct an overall survey on the surface structure of the samples of the media language by analyzing the formation mechanism of memes in media language and to determine their salient features.

The second step was to classify the data. The formation mechanism of media language meme has been classified on the basis of their linguistic forms in accordance with their specific contexts where the media language appears. However, in the process of the classification, it was noticed that some media language memes had more than one feature. In addition, the renovation of the media language was fast owing to the dynamic quality and randomness of their variation. For these reasons, the classification was rather difficult, and the data collection was impossible to cover all aspects of media language.

The third step was to analyze the data to explore the deep-rooted reasons and motivations for the creation of media language memes, probing into drives that make the media language replication and transmission, in particular, explaining how media language memes become strong and productive during the evolution.

\section{RESULTS AND DISCUSSIONS}

\subsection{Analysis of Memetic Phenomena of media language}

Based on the analysis of the data, it is revealed that the formation of media language memes can't take shape without their own practicality. With the popularity of a variety of media platforms, media language is used by more and more media audience and some language items become language memes due to their own specific meanings and characteristics. Next, rationality is one of the characteristics of media language memes. Thirdly, fashion is another characteristic of media language memes. Finally, if the language information has been uttered by some authorities, it will be constantly replicated and widely transmitted.

In addition to the abovementioned characteristics, media language memes also have their unique features like conciseness, innovativeness, vividness.

Above all, most of people like to use the briefest words and expressions to transmit information because concise memes can save time. Therefore, conciseness becomes one of the main features of media language memes. For example, people often use acronyms or figure homophone, such as "PK" (Playing Killing), "F6" (It stems from the Chinese pronunciation of "I admire you"). Those language memes are gradually accepted and used in the platform of the Internet in particular.
Secondly, one of the most impressive characteristics of media language memes is innovativeness, and many utterances rarely occur in daily life. Enormous creative expressions can be found on all the platforms of media past and present. The novel memes are readily recognizable and appeals to more and more audience. At last, language expression of media memes is also vivid. The memes possess a very strong sense of imagination and are extremely vivid, which greatly improves a sense of humor.

According to the analysis of the data, in the process of replication and transmission, the content and form of media language memes are not always identical between the original one and the replica. Moreover, media language memes have different ways of replication and transmission. Based on the classification proposed by $\mathrm{He}$ (2004) and the analysis of the data, the replication and transmission ways of media language memes are summarized as follows:

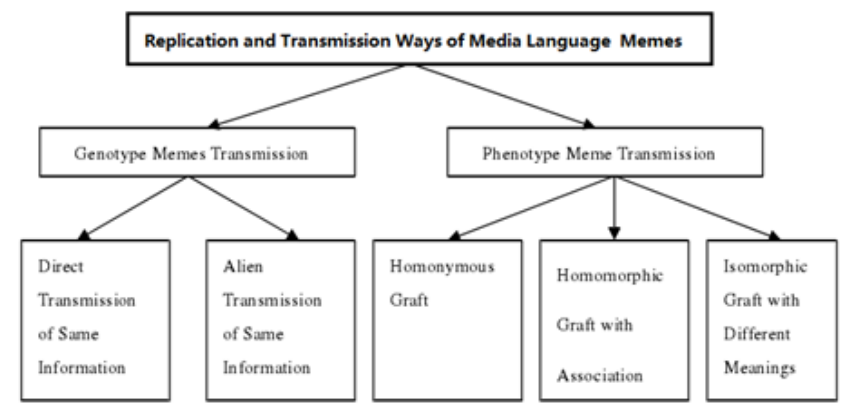

Figure 1 Replication and Transmission Ways of Media Language Memes

\subsection{Adaptation of Media Language Memes to Contextual Correlates}

Main contextual correlates of adaptability for media language memes involve mental world, social world of communicators and the media's characteristics (Verschueren, 2000). The mental world activated in language use encompasses cognitive and emotive factors (Verschueren, 2000).

Based on the analysis of the data, it is identified that the media language memes adapt to the cognitive elements in their transmission. According to Blackmore (2000), the reason why successful memes could spread themselves lies in that they are memorable. Memorization process encompasses encoding, storage and retrieval of information and they form a system called working memory (Nelson, 2008). Hence, in order to gain the opportunity to be retrieved, the media language memes are always capable of being stored in the working memory actively.

In the analysis process of the data, it is also noticed that the media language memes adapt to the emotional elements in its transmission. According to Blackmore (2000), certain memes almost force their hosts to transmit them on. In fact, emotion is the 
driving force for media language memes which contains information like useful instructions or juicy scandals to travel in the life circle. Emotional factors exert significant influence on the selection and transmission of media language memes. They adapt to emotional factors of human beings to gain competitive advantages over other language memes.

During the analysis of the data, it is discovered that media language memes also adapt to the social world. Numerous factors influence the choicemaking of language use, and the adaptation process is complicated and dynamic. According to the previous studies (Liu 2007 Verschueren 2000, Wang 2013 among others), most of the social environment factors and social norms affect the behavior of language users. For example, social position, race, religion, gender, age, culture and so on greatly affect linguistic choice making.

More specifically, it is shown that different media language memes adapt to the characteristics of different media platforms. For example, netspeak meme choices in the propagation and replication process are found to comply with the contextual elements of the Internet (Wang, 2013). In addition, according to Liu (2007), news language memes adapt to the standardization of journalism language, with the result of building up authority of the news reports.

\section{CONCLUSIONS}

This paper explores the characteristics and classifications of media language memes and investigates their adaptation to the contextual correlates (e.g. mental world, social world) in the process of transmission and replication.

However, there is limitation in this study. One limitation of this research is that the collection of data could not cover all memetic adaptation phenomena of the media language. Secondly, media language is global including many languages, while data collection in this study mainly focuses on Chinese and English. Therefore, the question whether the process of memetic adaptation of media language is the same in different languages needs to be further explored. Finally, contextual correlates in adaptation theory are very complicated and different media language memes might come down to different contextual correlates. However, this paper discussed and analyzed only three factors rather than all elements in the memetic adaptation process.

Memetic adaptation provides a new perspective for media language research and communication. Therefore, the research along this line should be further carried out in the future. According to the abovementioned limitation, individual meme might be discussed in terms of its all contextual factors in details. Besides, the data collection should involve more languages to conduct comparative study in this field. In a word, it is of significance to further explore the abovementioned aspects in future study.

\section{REFERENCES}

[1] Aitchison, J. and Lewis, D 2003 New Media Language Routledge

[2] Atran, S. 2001. The trouble with memes: Inference versus imitation in cultural creation. Human Nature 12 (4), 351381.

[3] Blackmore, S. 2000.The power of memes Scientific American, (4): 52-61

[4] Brodie, R. 1996. Virus of the Mind: The New Science of the Meme. Seattle: Integral Press.

[5] Dawkins, R. 1976. The Selfish Gene (2nd edition 1989). Oxford: Oxford University Press.

[6] Heath,C. and Seidel, V. 2004 Language as a Coordinating Mechanism: How Linguistic Memes Help Direct Appropriate Action http://www.si.umich.edu/ICOS/Linguisticmemes4.2.pdf

[7] Liu, J. (2007) A Memetic-adaption Approach to the Study of the Popularity of News Headlines (MA thesis)

[8] Moritz, E. 1990. Memetic science: I general introduction. Journal of Ideas (1), 1-23.

[9] Nelson, C. 2008 What are the differences between longterm, short-term, and working memory? Prog Brain Res. 169 (169): 323-338.

[10] Verschueren, J. 2000. Understanding Pragmatics Beijing: Foreign Language Teaching and Research Press

[11] Wang, L. 2013 A Study on Adaptation of the Netspeak Meme Phenomenon (MA thesis) 